\title{
Cross-Cultural and Socioeconomic Influences on Urological Cancer Patients
}

\author{
Sabina Cerimagic \\ The Faculty of Engineering and Information Technology and the Medical School, The University of Sydney, Australia \\ *Corresponding Author: Sabina.cerimagic@sydney.edu.au
}

Copyright (C) 2013 Horizon Research Publishing All rights reserved.

\begin{abstract}
This journal paper is a literature review which aims to take a closer look at how different ethnicities/races, cultures and socioeconomic status affect both patients and their families and how those factors influence patients' medical decision-making. Both culture and socioeconomic status have been shown to influence the stage at which an individual is diagnosed, how that individual copes with their diagnosis and what medical decisions the patient will make. Hence, it is of utmost importance that more research in the area of cross-cultural and socioeconomic influences on urological cancer patients is conducted. Additionally, medical practitioners must take into consideration patients' cultural and socioeconomic backgrounds when treating them if the treatment is to be effective and efficient. A lack of cultural and socioeconomic understanding by doctors may lead to misperceptions and misunderstandings, which in turn can lead to poor interaction between patients and doctors as well as unwanted or inappropriate clinical outcomes.
\end{abstract}

Keywords Urological Cancers, Socioeconomic Status Cross-Culture, , Medical Decision Making, Minority Health, Socio-Cultural Barriers, Multicultural Health

\section{Introduction}

At the time of writing this paper the influences of cross-cultural and socioeconomic factors on urological cancer patients have not been researched. Additionally, most of the existing research that has been done on the cross-cultural and socioeconomic influences on cancer patients has been based on studies from the United States (US), where the racial and ethnic groups most commonly studied and compared are African Americans and non-Hispanic white Americans [1] [2].

Physicians encounter patients from diverse cultural and socioeconomic backgrounds and those patients have various beliefs, values and behaviours regarding health. Research into patient ethnicity has shown ethnical disparities in health, with minority groups suffering disproportionally from cancer and other serious medical conditions [3] [4] [5].
Additionally, members of minority ethnic groups often have lower levels of education, work in jobs that have greater occupational hazards, live in areas with more environmental hazards and come from lower socioeconomic backgrounds than the majority population [5]. Ethnic differences and socioeconomic influences play a major role in cancer diagnosis and treatment as different cultures have different belief systems and socioeconomic influences.

This literature review takes a closer look at urological cancers and describes what existing research finds about which urological cancers are more or less common in certain ethnicities. Moreover, a brief definition of culture and ethnicity is provided and the cross-cultural and socioeconomic influences on cancer patients are identified and described. Each individual is influenced by his or her culture and patients' views on cancer can affect how the patient and the patient's family will react to cancer. Culture as well as patients' socioeconomic background will have an effect on patients' health decision-making and the psychological and emotional support they will request or use.

\section{Materials and Methods}

This journal article is an academic literature review of cross-cultural and socioeconomic influences onto urological cancer patients. It was conducted by searching the PubMed and EBSCO databases as well as Google scholar for the following keywords: socioeconomic influences on health, cultural influences on heath, race/ethnicity and urological cancer, prostate/bladder/kidney/testicular cancer and culture/socioeconomic influences, culture and medical decision-making, cancer diagnosis and fatalism, minority health, sociocultural barrier, multicultural health, both alone and in combination. Only publications that specifically addressed cross-cultural and socioeconomic influences on prostate, bladder, kidney and/or testicular cancers were included in this review.

\subsection{Urological Cancers}


Cancer that affects the prostate, bladder, kidneys and testicles are known as urological cancers.

Prostate cancer is highly heterogeneous in both disease and progression and is the most common male cancer in the western world [6]. The highest rates of prostate cancer are found in the United States, Canada, Scandinavia and Australia and the lowest rates are found in China and other parts of Asia [7] [8] [9] [10] [11]. According to researchers such as Crawford [12] and Smedley, Stith and Nelson [13], these differences are caused by genetic susceptibility, ethnicity, cultural influences, socioeconomic factors, exposure to unknown external risk factors and differences in health care and cancer registration, either singly or in combination.

Bladder cancer is more common in males then in females and its incidence increases with age [14]. The incidence of bladder cancer varies significantly amongst countries, with the highest incidence rates occurring in Australia and Eastern European counties where smoking in men is prevalent and parts of North America. The lowest rates of bladder cancer occurrence are found in Far Eastern countries [15] [16]. Since the early 1980s it has been known that bladder cancer can be caused by chronic infection with Schistosoma haematobium, which is common in parts of the Middle East and Africa. Occupational exposure and cigarette smoking have also been linked to bladder cancer. Additionally, research that has been conducted primarily in the US indicates that bladder cancer rates are higher in Caucasian/non-Hispanic Caucasians than in African Americans [16]. This information was one of the first discoveries in cancer research, as bladder cancer and lung cancer were among the first cancers to undergo in-depth epidemiological investigations [14].

Among urological cancers, kidney cancer is the third highest in incidence, following prostate carcinoma and transitional cell carcinoma of the bladder [17]. Kidney cancer occurs more frequently in urban populations than in rural ones, with the highest rates of incidence found in Denmark, Australia, New Zealand, North America and parts of Europe. The lowest incidences of kidney cancer are in Spain, Ireland, Italy, Japan, Venezuela, India and China [16] [18]. This may be due to a number of factors, such as the smoking habits of individuals living in those countries, chemical carcinogens, viruses (such as HIV infection), inherited traits (for example genetic or racial), diuretics, diet and obesity and acquired cystic disease/chronic dialysis in urban populations [17]. However, according to McLaughlin, Blot and Devesa [19] there is no relation between the socioeconomic or educational status of patients and a diagnosis of kidney cancer.

Testicular cancer (TC) is the most common cancer in 20 to 34-year-old men in western countries. Numerous publications have shown that there are substantial differences in occurrence rates in testicular cancer among countries, and since the 1980s there has been an increase incidence of testicular cancer [20]. Testicular cancer incidence rates have been uniformly increasing in many industrialised populations, with the most steep and consistent increases found among populations with European, Australian, New Zealand and North American ancestry [21] [16]. According to Swerdlow, Douglas, Huttly and Smith [22] testicular cancer risk is greater in men of high socioeconomic status and the risk has been found to be highest in men in professional and managerial professions. Unlike the other three urological cancers, testicular cancer is poorly understood. Sincic, Kulis, Znao and Bray [23] find that Slovenia and Croatia have one of Europe's highest incidence rates of testicular cancer, yet it remains largely unknown why rates are so much higher in those regions. Awareness of why testicular cancer occurs more frequently in some countries may assist in understanding the causes for testicular cancer in general. Future epidemiological studies may shed some light on why curtain ethnicities have higher rates than others of being diagnosed with the illness.

It is important to understand which races and ethnicities have the highest risk of being diagnosed with one or more of the urological cancers so that the diagnoses of these diseases is timely and the treatment is effective and efficient. Currently there are no cancers with poor prognostic molecular markers or clear genetic differences that correlate with more aggressive cancers in African Americans than Caucasian Americans. However, there are other factors that can influence the incidence of cancer, for example not seeking medical help soon enough because the patient comes from a lower socioeconomic background and cannot afford health insurance. The patients' culture, level of education and understanding of their illness will affect their attitude towards their diagnosis and treatment. For example, the stage and influence of urological cancers might be influenced if the patient thinks that being diagnosed with one or more of the urological cancers is a terminal prognosis and therefore believes there is no point in seeking any further help.

\subsection{A Brief Definition of Culture and Ethnicity}

Providing a conclusive and general definition of culture is considered impossible by cultural scholars and often leads to controversy in trying to define what exactly constitutes culture [24]. The culture of an individual is influenced by the region and society where an individual grows up, as well as other factors that affect daily life such as education, language and socioeconomic status. Each individual's experiences are different and they all contribute to what each individual defines as their 'culture'. The culture of a society consists of the elements of each of the individuals within the society. Hence, a culture cannot be summarised and limited into one single characteristic, because its meaning is diverse and different for each individual, society and area of application. Although a culture is made up of individuals who are different, culture influences behaviour and provides explanations for how a group of people with the same cultural background communicate and filter information [25]. However, culture, like language, does not provide a blueprint for a group's behaviour [26]. Every individual is influenced 
by and belongs to a number of cultures that include but are not exclusive to race and ethnicity. In the literature, the terms ethnicity and race are used interchangeably. Ethnicity refers to a person having an ethnic quality or affiliation to an ethnic group. Al-Atiyyat [26] defines an ethnic group as a large group of people that have collective customs or traits in common. Race, however, is used more precisely to refer to traits that are transmissible by descent and distinguishing characteristics that identify a racial group [26].

Considering ethnicity and race is important when diagnosing cancers, as some minority groups are more predisposed to certain illnesses than others. Davis et al. (2001) notes that there is a disparity in tumour grade between African-American patients and Caucasian Americans, with African Americans presenting with higher-grade tumours than Caucasian Americans. This is very often linked to African-American patients having an inadequate level of education and a lower income and socioeconomic status than Caucasian Americans, and therefore not being able to afford health insurance. The socioeconomic status of an individual will also have an influence on his or her health decision-making. In countries like the US, prostate cancer patients from minority groups, for example African Americans, are often diagnosed with more advanced-stage cancers then Caucasian non-Hispanic Americans. This is often linked to the African-American patients not being able to afford health insurance. Uninsured patients have less access to health care and preventative services than those who have health insurance [27].

\subsection{The Symbolic Meaning of Cancer}

How a patient views cancer has an effect on how the patient and the patient's family will react to cancer. A lot of research has been done on views on cancer in Western culture, which has found that Anglo-Americans tend to view illness as an unnatural part of life and believe that cancer is brought on by external factors over which the patient has no control. Western medicine also focuses on interventions and cancer is viewed in a mechanistic fashion, which means that cancer needs to be treated with proper Western medicine [28] [29]. In Asian culture, cancer is seen as being brought on by the individual through lifestyle choices, genetic predisposition and by weakness of character [30]. Additionally, in Asian culture some cancers, especially cancers such as prostate cancer, testicular cancer and breast cancer, have a social stigma attached to them and seeking medical help and support outside one's immediate circle is discouraged. Asian practices and values do not encourage the direct communication of emotional feelings and family cohesiveness and conformity are fundamental [31] [32] [33].

Asian cancer patients believe that directly seeking help from family and friends is seen as complaining. This belief can also prevent an individual from seeking medical help. Meeting adversity without complaining is a sign of dignity and asking others for help is seen as being demanding and a lack of character [29]. Due to those cultural barriers, an
Asian cancer patient is less likely than a Western patient to seek help from mental health services. An Asian patient's culture teaches them that they have to be self-sufficient and if they cannot cope with (for example) their bladder cancer, they are to only ask for help within their own family. Seeking help from outside the family would be seen as disgraceful, shameful and demonstrating weakness of character [29] [34].

Nathan [35] found that there are striking ethnic differences in both the cancer incidence and mortality rates in American men. Targonski [36] believe that the stage of diagnosis and mortality are due to ethnic differences. The research of Hoffman [27] illustrates that lower socioeconomic status individuals and individuals who are unemployed or underemployed across all races usually had an advanced-stage prostate cancer at the time of diagnosis. This indicates that not only culture but also the socioeconomic status of an individual contributes to how and when they are diagnosed and treated for their disease. A Quality of Life (QOL) study conducted by Whaley and Davis [37] found that cultural factors must be considered when diagnosing patients and when evaluating the treatment and outcomes of cancer patients, since those cultural values can affect many facets of an individual's life. There are many ways that culture impacts upon the diagnosis and treatment of cancer. In some cultures, asking for emotional support outside of the family is not encouraged and it is strongly frowned upon [29] [34]. According to Rasheed [38], ethnic difference affects knowledge about prostate cancer and increases the chance of fatalism once diagnosed with the illness and poor adherence to prostate cancer screening, all of which can result in patients being diagnosed with the illness at a more advanced stages. The likelihood of being screened for prostate cancer is influenced the patient's socioeconomic status and their knowledge about the illness and the health benefits of screening [35]. This highlights a need to educate the public about serious illnesses like cancers, as some, in particular lower socioeconomic groups, are at a particular disadvantage regarding knowledge of those illnesses.

\subsection{Cross-Cultural and Socioeconomic Influences on Cancer Patients}

Biological, cultural, social and socioeconomic factors influence cancer incidence, treatment and outcomes in patients [28]. Yarbo [39] believe that between 80 and 90 per cent of cancers are lifestyle-dependent. Kagawa-Singer [28] states that even cancers that have a genetic predisposition sometimes require environmental exposures to ontogenesis the process. An example of this is demonstrated by [40], who studied Helicobacter pylori and found a correlation between Japanese-American men in Hawaii who were diagnosed with the bacteria and the development of gastric carcinoma. Additionally, migrants who move from countries with low incidences of prostate cancer to countries with a higher risk show an increase in incidence; a general example of this is a Japanese man who moved to the US. The increase in incidence of prostate cancer could be due to environmental 
changes such as diet [8] [16]. A patient's culture is correlated with race and ethnicity [28] as well as a much wider range of factors such as higher smoking rates, inadequate education, unemployment, substandard housing, psychological stress and other environmental factors. For example, inadequate education is usually a characteristic of low socioeconomic individuals who in turn have an attitude of fatalism. Patients who have an attitude of fatalism believe that death is unavoidable, which means that a patient who receives a negative medical result, such as being diagnosed with prostate cancer, may think there is no hope and the end is near [41]. This was demonstrated in Loeher [42] study of 128 cancer patients from lower socioeconomic backgrounds, which reveals that due to lack of education almost 50 per cent of those patients did not know that smoking can cause cancer. Additionally, African Americans, who usually come from lower socioeconomic backgrounds, often have more fatalistic views of cancer and have lower rates of screening then non-Hispanic white Americans [43].

Individuals from lower socioeconomic backgrounds often have limited education [44]. Understanding the role of education in the timely diagnosis of cancer is important as, for example, bladder cancer has been associated with a number of factors, such as smoking [45] so the patients' cultural background plays a role in their cancer diagnosis. Nomura [40] study examined bladder cancer patients from Caucasian and Japanese backgrounds living in Hawaii who smoke. They noticed that males from both those ethnic groups who smoked had a significantly elevated risk of bladder cancer compared to those who did not smoke.

Researchers including Biggs [46] Gajendran [47] Hu [48] Lantz [49] and Oakley-Girvan [50] highlight that lower socioeconomic factors as well as patient ethnicity are associated with the presentation of advanced-stage cancers at diagnosis. According to Halpern [51] research is of utmost importance to address the cultural and social factors that may limit access to cancer screening and early detection. Halpern [51] highlight that ethnicity is a significant predictor of more advanced-stage disease at diagnosis.

\subsection{Psychological, Emotional and Support Issues}

Once diagnosed with cancer, most individuals will experience an array of emotions, ranging from confusion to depression and even denial. Being diagnosed with cancer can be highly stressful, not only for the patient but also for the patient's family and sometimes even friends. Friends and family of cancer patients can find dealing with the illness more stressful then the patients, as family members feel hopeless and uncertain of how best to provide assistance [33]. Research indicates that spouses find it very hard to deal with the burden of diagnoses and associated stress and often experience anxiety and depression [33] [43]. In a US study, Gore [52] found that marital status is related to cancer survival and their research indicates that Caucasians, Hispanics and Asian Pacific Islander (API) patients have 20 per cent higher marriage rates than African Americans.
Those researchers found that patients who were married would receive psychosocial support from their spouses, who would also insist that the patient undergoes regular medical check-ups and would even help with earlier diagnosis by encouraging the patient to go see a doctor to evaluate symptoms. The spouses may also insist that the patient obtain more than one medical opinion, go to higher-quality facilities for treatment, stop drinking alcohol and smoking and improve their diets.

The aetiology of cancer is multifunctional; it is for this reason that it is important to recognise a patient's ethnic background and their socioeconomic status, as these two factors influence patients' attitude and behaviour. For example, a patient from a lower socioeconomic background may not be able to get the same family support as someone who is more financially secure. Most cancer patients need ongoing and extended treatment and this has financial, physical and social impacts on the patients and their families. The medication or treatment that the patient requires may be expensive, transportation maybe not be available and the patient's family members may not be able to take time off work to care for the patient. According to Given [53] the support or lack of support a patient receives from his or her family greatly influences the patient's quality of life, clinical outcomes and even survival.

\subsection{Medical and Emotional Help-Seeking Behaviours}

In their pilot study, Campbell [54] finds that Caucasian men experience better prostate cancer outcomes than African-American men. African-American men not only have higher prostate cancer incidence and mortality rates than Caucasian men, they also cope less well with the side effects of treatment. Following acute recovery from treatment, prostate cancer survivors often still need to cope with both the emotional and physiological symptoms that remain long after treatment [55] [56]. Researchers such as Lubeck [57] Campbell [54] and Rosenthal [58] stress that African-American prostate cancer patients recover more slowly from treatment then Caucasian prostate cancer patients and they also have a lower quality of life related to both physical and physiological symptoms. Bloor [59] find that patients who have a better social support system are positivity influenced by it, in that those patients had a better perception of their physical health.

African-American prostate cancer patients are unlikely to seek physiological services as there is a social stigma attached to those services and a belief that using them is a sign of weakness [60] [61]. Research into breast cancer indicates that female cancer survivors are far more likely than male cancer survivors to attend support groups and ask for emotional help [62 - 64]. Chambers [65] also notes that men are underrepresented as clients at cancer services and that there are much less likely then woman to seek help for psychological issues such as depression and anxiety. Jayadevappa [66] highlight that there have been a number of studies that indicate that race and ethnicity are very 
important precursors of both treatment and outcomes for prostate cancer. However, since most existing research focuses primarily on the US help-seeking patterns for depressive disorders; little data is available on cultures outside the US [67].

Rickwood and Braithwaite [68] completed an Australian study that analysed emotional help-seeking behaviours in 715 adolescents. They find that male teenagers do not like to ask for help, either from their social networks, such as family and friends, or from professionals. Other studies note that, culture can have an effect on the help-seeking behaviour of men as well. In some cultures, such as the African-American culture, seeking help from mental health professionals is seen as a sign of weakness. In Middle Eastern and Asian countries, it is not only seen as a sign of a weak character but it also could bring shame onto the family. Therefore, seeking help outside the family is not encouraged.

Ethnic differences and socioeconomic factors both have a major impact on cancer diagnosis and treatment. Different cultures have different belief systems and socioeconomic influences will guide how much and the quality of medical/emotional help-seeking a patient and his or her family can afford.

\section{Conclusion}

Urological cancers have not been studied simultaneously before and at the time of writing this paper the cross-cultural and socioeconomic influences on urological cancer patients have not been researched. Culture is a vital part of every individual's life and accordingly it plays an important part in the context in which people make decisions about how to act. Every individual is influenced by a number of factors, such as their gender, age, personal psychology, religion and socioeconomic status. These cultural and socioeconomic influences will be major influences on a patient's medical decision-making. If, for example, prostate cancer patients from a particular socioeconomic or cultural group feel that there is a stigma attached to seeking emotional assistance, doctors need to be aware of this so they know to explain the benefits of those services to patients and therefore help them move past the stigma. This is only one example, but it outlines that if the doctor has a better understanding of patients' cultural and socioeconomic backgrounds, they will be able to find ways in which they can effectively help the patients.

To successfully help patients with terminal illnesses such as urological cancers, physicians need to understand the cultural and socioeconomic influences that affect patient's beliefs, behaviours and medical decision-making. To date little attention has been paid to research on the cross-cultural and socioeconomic influences on cancer patients. Additionally, at the time of writing this paper there is no research that investigates the cross-cultural and socioeconomic influences on urological cancer patients.

\section{Acknowledgements}

We are very grateful to Professor Suzanne Chambers, from Griffith University (QLD, Australia) for taking the time to read the manuscript and provide us with constructive feedback.

\section{REFERENCES}

[1] A. Jemal, A. Thomas, T., Murray, et al. Cancer statistics, 2002. CA Cancer J Clin, 52, 23-47. 2002

[2] A. Jemal., L.X. Clegg, E. Ward, E., et al. Annual report to the nation on the status of cancer, 1975-2001, with a special feature regarding survival. Cancer, 101, 3-27. 2004.

[3] A. Antonovsky, A. Social class and the major cardiovascular diseases. Journal of Chronic Disease, 21(2), 65-106. 1968.

[4] D. R. Williams \& T. D. Rucker. Understanding and addressing racial disparities in health care. Health Care Financ Rev. 21(4), 75-90. 2000.

[5] J. R. Betancour. Cross-cultural Medical Education: Conceptual Approaches and Frameworks for Evaluation. 560 Academic Medicine, 78, (6). 2003.

[6] J.C. Chambers. P. Elliott. D. Zabaneh. W. Zhang. Y. Li P. Froguel. D. Balding. J. Scott. \& J.S. Kooner. Common genetic variation near MC4R is associated with waist circumference and insulin resistance. Nat Genet, 40, 716-718. 2008.

[7] D. M. Parkin, \& C. S. Muir. Comparability and quality of data. In: , D.M. Parkin, C.S. Muir, S.L.Whelan, Y.T. Gao, J. Ferlay \& J. Powell (eds), Cancer Incidence in Five Continents, Volume VI (IARC Scientific Publications No. 120), Lyon, IARC. 1992.

[8] D. M. Parkin, F. Bray, J. Ferlay, P., Pisani. Global cancer statistics, 2002. CA Cancer Journal Clinical, 55, 74-108. 2005.

[9] D. M. Parkin, V. W. Chen, J. Ferlay, J. Galceran, H.H. Storm, \& S.L. Whelan. Comparability and Quality Control in Cancer Registration (IARC Technical Report No. 19), Lyon, IARC. 1994.

[10] M. Quinn, \& P. Babb.Patterns and trends in prostate cancer incidence, survival, prevalence, and mortality. Part International comparisons, BJU Int, 90, 162-173. 2002.

[11] H. Gronberg. Prostate cancer epidemiology. Lancet, 361, 859-864. 2003

[12] E.D. Crawford. Epidemiology of prostate cancer. Urology, 62, 3-12. 2003.

[13] B. Smedley, A.Y. Stith, \& A.R. Nelson. Unequal treatment: confronting racial and ethnic disparities in health care. Washington, DC: The National Academies Press, 764. 2003.

[14] M.P. Zeegers. E. Kellen. F. Buntinx, \& P.A. van den Brandt. The association between smoking, beverage consumption, diet and bladder cancer: a systematic literature review. World J. Urol., 2(1), 392-401. 2004.

[15] B.W. Steward, \& P. Kleinhaus. World Cancer Report: WHO-IARC, Lyon. 2003. 
[16] D.M. Parkin, P. Pisani, \& J. Ferlay. Global Cancer Statistic. Cancer Journal for Clinicians, 49(1). 1999.

[17] D. Pascual, \& A. Borque. Review Article Epidemiology of Kidney Cancer Hindawi Publishing Corporation, Advances in Urology, Article ID 782381, 7 pages. 2008.

[18] S.L. Parker, T. Tong, S. Bolden, \& P. A. Wingo. "Cancer statistics, 1997," Ca: A Cancer Journal for Clinicians, 47(1), 5-27. 1997.

[19] J.K. McLaughlin, W.J. Blot, \& S.S. Devesa. "Renal cancer," in Cancer Epidemiology and Prevention, D., Schoffendeld and J. F., Fraumeni Jr., Eds, 1142-1155, Oxford University Press, New York, NY, USA, 2nd edition. 1996.

[20] E. Huyghe, T. Matsuda, and P. Honneau. Review Article, Increasing Incidence of Testicular Cancer Worldwide: a review. The Journal of Urology, 170, 5-11. 2003.

[21] M.P. Purdue, S.S. Devesa, A.J. Sigurdson, \& K.A. McGlynn. International patterns and trends in testis cancer incidence. International Journal of Cancer, 115, 822-827. 2005.

[22] A.J. Swerdlow, A. J., Douglas, S.R.A. Huttly, \& P.G. Smith. Cancer of the testis, socioeconomic status, and occupation. British Journal of Industrial Medicine, 48, 670-674. 1991.

[23] N. Sincic, T. Kulis, A. Znaor, F. Bray. Time trends in testicular cancer in Croatia 1983-2007: rapid increases in incidence, no declines in mortality. Cancer Epidemiol, 36(1), 11-15. 2012.

[24] J.K. Cowan. Culture and Rights after Culture and Rights. American Anthropologist, 108(1), 9-24. 2006.

[25] A. Murphie, \& J. Potts. Culture and Technology, New York: Palgrave Macmillan.2003.

[26] N. M. H. Al-Atiyyat. Cultural Diversity and Cancer Pain. Journal of Hospice and Palliative Nursing , 11(3). 2009.

[27] C. Hoffman, et al. Medical Debt and Access to Health Care, Kaiser Commission on Medicaid and the Uninsured. 2005

[28] M. Kagawa-Singer, \& L.J. Blackhall. Negotiating Cross-Cultural Issues at the End of Life: "You Got to Go Where He Lives." JAMA, 286(23), 2993-3001. 2001.

[29] D. Wellisch, M. Kagawa-Singer, S.L. Reid, Y. Lin, S. Nishikawa-Lee, \& M. Wellisch. An exploratory study of social support: a cross-cultural comparison of Chinese-, Japanese-, and Anglo-American breast cancer patients. John Wiley \& Sons, Ltd. 1999.

[30] R.F. Murphy. The Silent Body, W.W. Norton, New York. 1990.

[31] L.L. Northouse. Breast cancer in younger women: Effects on interpersonal and family relations. Journal of the National Cancer Institute Monographs, 16, 183-190. 1994.

[32] L.L. Northouse, G. Dorris, \& C. Charron-Moore. Factors affecting couples' adjustment to recurrent breast cancer. Social Science and Medicine, 41, 69-76. 1995.

[33] L.L. Northouse, M. Jeffs, A. Cracchiolo-Caraway, L. Lampman, \& G. Dorris. Emotional distress reported by women and husbands prior to a breast biopsy, Nursing Research, 44, 196-201. 1995.
[34] L. Uba. Asian American: Personality Patterns, Identity, and Mental Health, Guilford Press, New York, 196-213. 1994.

[35] S.C. Nathan A. H. Morgenstern, E. Kudadjie-Gyamfi, C. Magai, \& A.I. Neugut. Prostate Cancer Screening Behavior in Men from Seven Ethnic Groups: the Fear Factor. Cancer Epidemiol Biomarkers, 15, 228-237. 2006.

[36] P.V. Targonski, P. Guinan, \& C.W. Phillips. Prostate cancer: the stage disadvantage in the Black male. J Natl Med Assoc, 83, 1094-6. 1991.

[37] T.C. Davis, N. Dolan, \& M.R. Ferreira, et al. The role of inadequate health literacy skills in colorectal cancer screening. Cancer Invest, 19, 193-200. 2001.

[38] M.A. Rasheed. The Influence of Cancer Fatalism and Prostate Cancer Knowledge on Sexual Function and Bother after Treatment for Prostate Cancer: A Moderation Model to Explain Ethnic Differences, Open Access Dissertations, Paper 853. 2012.

[39] J.W. Yarbro. Milestones in our understanding of the causes of cancer. In: Groenwald SL, Frogge MH, Goodman M, et al. editor. Cancer Nursing Principles and Practice. ed 3. Boston, MA: Jones and Bartlett, 1993:28-46. 1993.

[40] A. Nomura, G.N. Stemmerman, \& P.H. Chyou, et al. Helicobacter pylori infection and gastric carcinoma among Japanese Americans in Hawaii. $\mathrm{N}$ Engl J Med, 325, 1132-1136. 1991.

[41] B. Powe, \& R. Finnie. Cancer fatalism: The state of the science. Cancer Nursing , 26 (6), 454-465. 2003.

[42] P.J. Loeher, H.A. Gregar, M. Weinberger, et al. Knowledge and beliefs about cancer in a socioeconomically disadvantaged population. Cancer, 68, 1665-1671. 1991.

[43] M.A. Debb, M.A. Blitz\& S.W. Choi. Research Report Quality of Life Differences In an African American and Caucasian Sample of Chronic Illness Patients: Assessment of Differential Item Functioning. The New School Psychology Bulletin, 6, 1. 2009.

[44] K. Espinosa de los Monteros, \& L. Gallo. The relevance of fatalism in the study of Latinas' cancer screening behavior: A systematic review of the literature. International Journal of Behavioral Medicine. 2010.

[45] H. Anton-Culver, A. Lee-Feldstein, \& T.H.. Taylor. The association of bladder cancer risk with ethnicity, gender, and smoking. Epidemiol,n3, 429-433. 1993.

[46] M. L Biggs. \& S. M. Schwartz. Differences in testis cancer survival by race and ethnicity: a population-based study, 1973-1999 (United States), Cancer Causes Control, 2004:15, 437-444. 2004.

[47] V. Gajendran, M. Nguyen, \& L. Ellison. Testicular cancer patterns in African-American men. Urology, 66, 602-605. 2005.

[48] Q. Hu, C.W. Zhang, and M. Sommerfeld. Biodiesel from Algae: Lessons Learned Over the Past 60 Years and Future Perspectives, Juneau, Alaska: Annual Meeting of the Phycological Society of America, 40-41. 2006.

[49] P. Lantz, J. House, J. Lepkowski, D. Williams, R. Mero, \& J. Chen. Socioeconomic factors, health behaviors, and mortality, Results from a nationally representative prospective study of 
US adults. Journal of American Medical Association, 279(21), 1703-1708. 2006.

[50] I. Oakley-Girvan, L.N. Kolonel, R.P. Gallagher, A.H. Wu A. Felberg \& A.S. Whittemore. Stage at diagnosis and survival in a multi-ethnic cohort of prostate cancer patients. $\mathrm{Am} \mathrm{J}$ Public Health, 93, 1753-9. 2003.

[51] M.T. Halpern, E.M. Ward, A.L. Pavluck, N.M. Schrag, J. Bian, \& A. Y. Chen. Association of insurance status and ethnicity with cancer stage at diagnosis for 12 cancer sites: a retrospective analysis. Lancet Oncol, 9, 222-231. 2008.

[52] J.L. Gore, C.S. Saigal, J.M. Hanley, M. Schonlau, \& M.S. Litwin. Variations in reconstruction after radical cystectomy, Cancer. American Cancer Society, 107(4), 729-737. 2005.

[53] B.A. Given. Family home and supportive care for breast cancer: What is the cost?. In: Paper presented to President's Cancer Panel Special Commission on Breast Cancer. Altanta: GA. 1993.

[54] L.C. Campbell. F.J. Keefe. C..Scipio. D. C. McKee. C. L. Edwards, S.H. Herman, L.E. Johnson. O.M. Colvin. C. M. McBride. \& C. Donatucci, C.. Facilitating research participation and improving quality of life for African American prostate cancer survivors and their intimate partners, A pilot study of telephone-based coping skills training, Cancer, 109:2, Article first published online: 15 DEC 2006.

[55] J.W. Saranchuk, M. W. Kattan, E. Elkin, A.K. Touijer, P.T. Scardino, \& J.A. Eastham. Achieving optimal outcomes after radical prostatectomy. Journal of Clinical Oncology, 23, 4146-4151. 2005.

[56] D.C, Miller, M.G. Sanda, \& R.L. Dunn, et al. Long-term outcomes among localized prostate cancer survivors: Health-related quality-of-life changes after radical prostatectomy, external radiation, and brachytherapy. J Clin Oncol, 23, 2772-2780. 2005.

[57] D.P. Lubeck, G. Grossfeld, P.S. Ray, S. Flanders. D. Penson, \& P.R. Carroll. Health-related quality of life (HRQOL) and sociodemographic profiles of African American men with prostate cancer: Data from CaPSURE. In: Program and abstracts of the American Urological Association 95th Annual Meeting, Atlanta, Georgia. Abstract 69.2000.

[58] D.S. Rosenthal. Changing trends: Cancer statistics, CA Cancer J Clin, 48, 3-4.1998.

[59] L. E. Bloor., R. S. Sandler. C. Martin, B.N. Uchino. \& A. Y Kinney. Associations between emotional support and health-related quality of life among a population-based sample of blacks and Caucasians. Journal of Social and Clinical Psychology, 25, 96-116. 2006.

[60] R.L. Meth, R. S. Pasick, eds. Men in Therapy: The Challenge of Change. New York: Guilford.1990.

[61] V.L. Sanders-Thompson, A. Bazile, M. Akbar. African Americans' perceptions of psychotherapists. Prof Psychol Res Pract, 35, 19-26. 2004.

[62] B. Weber, B. Roberts, \& GJJr. McDougall. Exploring the efficacy of support groups for men with prostate cancer. Geriatr Nurs, 21, 250-253.2000.

[63] B. Weber, B. Roberts, \& M. Resnick, et al. The effect of dyadic intervention on self-efficacy, social support, and depression for men with prostate cancer. Psychooncology, 13, 47-60.2004.

[64] B. Weber, B. Roberts, T. Mills, N. Chumbler, \& C. Algood. Physical and emotional predictors of depression after radical prostatectomy. American Journal of Men's Health, 2 (2), 165-171. 2008.

[65] S.K. Chambers, M. Ferguson, R. Gardiner, D. Nicol, L. Gordon, S. Occhipinti, \& J. Aitken. Study Protocol, ProsCan for Men: Randomised controlled trial of a decision support intervention for men with localised prostate cancer, BMC Cancer, 8, 207. 2008.

[66] R. Jayadevappa, J. Johnson, S. Chhatre, A. Wein, \& S. Malkowicz. Ethnic variation in return to baseline values of patient-reported outcomes in older prostate cancer patients. Cancer, 109 (11), 2229-2238. 2007.

[67] A.M. Möller-Leimkühler. Barriers to help-seeking by men: a review of sociocultural and clinical literature with particular reference to depression. Journal of Affective Disorders, 71(1-3), 1-9. 2002.

[68] D.J. Rickwood, \& V.A. Braithwaite. Social-psychological factors affecting help-seeking for emotional problems, Social Science \& Medicine, 39(4), 563-572. 1994. 